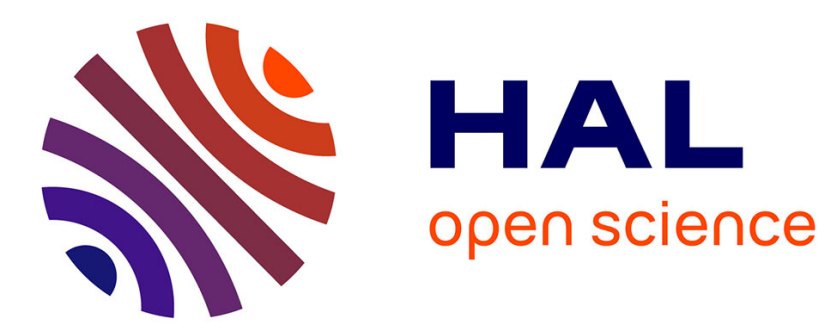

\title{
THE GAME OPERATOR ACTING ON WADGE CLASSES OF BOREL SETS
}

Gabriel Debs, Jean Saint Raymond

\section{To cite this version:}

Gabriel Debs, Jean Saint Raymond. THE GAME OPERATOR ACTING ON WADGE CLASSES OF BOREL SETS. The Journal of Symbolic Logic, 2019, 84 (3), pp.1224-1239. 10.1017/jsl.2019.40 . hal-02421644

\section{HAL Id: hal-02421644 https://hal.sorbonne-universite.fr/hal-02421644}

Submitted on 20 Dec 2019

HAL is a multi-disciplinary open access archive for the deposit and dissemination of scientific research documents, whether they are published or not. The documents may come from teaching and research institutions in France or abroad, or from public or private research centers.
L'archive ouverte pluridisciplinaire HAL, est destinée au dépôt et à la diffusion de documents scientifiques de niveau recherche, publiés ou non, émanant des établissements d'enseignement et de recherche français ou étrangers, des laboratoires publics ou privés. 


\title{
THE GAME OPERATOR ACTING ON WADGE CLASSES OF BOREL SETS
}

\author{
GABRIEL DEBS AND JEAN SAINT RAYMOND
}

\begin{abstract}
We study the behavior of the game operator $\partial$ on Wadge classes of Borel sets. In particular we prove that the classical Moschovakis results still hold in this setting. We also characterize Wadge classes $\boldsymbol{\Gamma}$ for which the class $\partial \boldsymbol{\Gamma}$ has the substitution property. An effective variation of these results show that for all $1 \leq \eta<\omega_{1}^{C K}$ and $2 \leq \xi<\omega_{1}^{C K}, \partial\left(D_{\eta}\left(\Sigma_{\xi}^{0}\right)\right)$ is a Spector class while $\partial\left(D_{2}\left(\Sigma_{1}^{0}\right)\right)$ is not.
\end{abstract}

\section{INTRODUCTION}

The game operator $D$ was extensively studied by Moschovakis in the context of adequate classes (see [7] for the definition). Let us only mention that an adequate class is closed under finite Boolean operation, and this seemingly mild closure property becomes extremely restrictive if one aims to cover general Wadge classes. As a matter of fact the only Wadge classes of Borel sets which are adequate are the Baire classes. The problem is that this closure property is used in a crucial way in most of the proofs in [7] and seems to be mandatory as far as one is working with "abstract" classes (see Section 5.2). However as we shall see, this assumption can be dropped if one restrict the study to Wadge classes of Borel sets. This is the main content of Theorem 5.5 from which one can prove all fundamental Moschovakis results such as the norm-transfer Theorem or the definable winning strategy Theorem for an arbitrary Wadge class of Borel sets.

Independently of this feature we also investigate the substitution property for $\partial \boldsymbol{\Gamma}$ classes when $\boldsymbol{\Gamma}$ is a given Wadge class. More precisely we prove that for a non self-dual class $\boldsymbol{\Gamma}$ the class $\partial \boldsymbol{\Gamma}$ has the substitution property if and only if either $\partial \boldsymbol{\Gamma}=\boldsymbol{\Pi}_{1}^{1}$, or $\partial \boldsymbol{\Gamma}=\boldsymbol{\Sigma}_{1}^{1}$, or $\boldsymbol{\Gamma}$ is of level $\geq 2$. Minor "effective" adaptations of these results provide new Spector classes. This is in particular the case for the classes $\partial\left(D_{\eta}\left(\Sigma_{\xi}^{0}\right)\right)$ for $1 \leq \eta<\omega_{1}^{C K}$ and $2 \leq \xi<\omega_{1}^{C K}$.

We mainly follow the notation and terminology of [7] with the exception of the notion of norm that we shall discuss later on in Section 6 .

By a class $\Gamma$ we shall always mean a class of pointsets closed under recursive substitutions and non trivial, that is $\Gamma$ as well as its dual class $\check{\Gamma}$ are not reduced to $\{\emptyset\}$, and as usual we define $\Delta(\Gamma)=\Gamma \cap \check{\Gamma}$. However we shall very often identify a given class $\Gamma$ to its relativization $\Gamma_{\omega^{\omega}}$ to $\omega^{\omega}$ and view thus $\Gamma$ as a subset of $\mathcal{P}\left(\omega^{\omega}\right)$.

2010 Mathematics Subject Classification. Primary Secondary .

Key words and phrases. Game operator, Wadge class, Norms, Scales, Substitution property. 


\section{WAdGe Classes of Borel SETS}

By a Wadge class we shall always mean a Wadge class of Borel subsets of $\omega^{\omega}$, that is a class of the form $\Gamma=\left\{\varphi^{-1}(U): \varphi: \omega^{\omega} \rightarrow \omega^{\omega}\right.$ continuous $\}$ for some Borel set $U \subset \omega^{\omega}$. Such a Borel set $U$ is then said to be generating $\boldsymbol{\Gamma}$. If $\boldsymbol{\Gamma}$ is non self-dual $(\boldsymbol{\Gamma} \neq \check{\boldsymbol{\Gamma}})$ then by determinacy, a Borel set $U$ generates $\boldsymbol{\Gamma}$ if and only if $U \in \boldsymbol{\Gamma} \backslash \check{\boldsymbol{\Gamma}}$. Also given any space $X \subset \omega^{\omega}$, we shall consider the relativization of $\boldsymbol{\Gamma}$ to $X$, that is the class $\boldsymbol{\Gamma}_{X}=\{B \cap X: B \in \boldsymbol{\Gamma}\}$.

For more on the description of Wadge classes we refer the reader to [5]. We only recall here some basic definitions and Folklore results needed for the sequel, and not easily found in the literature.

Proposition 2.1. Let $\boldsymbol{\Gamma}$ be a non self-dual Wadge class generated by a set $U$, let $X \subset \omega^{\omega}$ be a Borel set and suppose that $X=\bigcup_{n \in \omega} V_{n}$ and each $V_{n}$ is open in $X$. Then for a set $A \subset X$ the following are equivalent:

i) $A \in \boldsymbol{\Gamma}_{X}$

ii) $\forall n \in \omega, A \cap V_{n} \in \boldsymbol{\Gamma}_{V_{n}}$

iii) $A=\psi^{-1}(U)$ for some continuous function $\psi: X \rightarrow \omega^{\omega}$.

Proof. i) $\Rightarrow$ ii) is obvious.

ii) $\Rightarrow$ iii) Refining the open covering $\left(V_{n}\right)_{n \in \omega}$ if necessary we may suppose that each $V_{n}$ is a clopen subset of $X$ and $\left(V_{n}\right)_{n \in \omega}$ is a partition of $X$. Then for all $n \in \omega$, since $A \cap V_{n} \in \Gamma_{V_{n}}$ there exists a continuous function $\varphi_{n}: V_{n} \rightarrow \omega^{\omega}$ which reduces $A \cap V_{n}$ to $U$. Then glueing together the $\varphi_{n}$ we get a continuous function $\varphi: X \rightarrow \omega^{\omega}$ which reduces $A$ to $U$; hence $A \in \Gamma_{X}$.

iii) $\Rightarrow$ i) Set $U^{\prime}=\omega^{\omega} \backslash U \in \check{\Gamma} \backslash \boldsymbol{\Gamma}$ and consider the game in each run of which the players construct separately two reals in $\omega^{\omega}: x$ constructed by Player I, and $y$ constructed by Player II, and:

Player II wins the run $\Longleftrightarrow(x \notin X$ or $(x \in A \leftrightarrow y \in U))$

Note that any winning strategy for Player I provides a continuous function $\varphi: \omega^{\omega} \rightarrow X$ which reduces $U^{\prime}$ to $A$, hence $\psi \circ \varphi: \omega^{\omega} \rightarrow \omega^{\omega}$ is a continuous function which reduces $U^{\prime}$ to $U$, which is impossible since $U \notin \check{\Gamma}$. Hence Player II has a winning strategy which provides a continuous function $\theta: \omega^{\omega} \rightarrow \omega^{\omega}$ such that $X \cap \theta^{-1}(U)=A$, and since by definition $\theta^{-1}(U) \in \boldsymbol{\Gamma}$ then $A \in \boldsymbol{\Gamma}_{X}$.

We also recall the notion of level which will play a central role in our study.

Definition 2.2. Let $\pi=\left(P_{n}\right)_{n<N}$ be a countable partition of $\omega^{\omega}$. A set $A \subset \omega^{\omega}$ is said to be a $\pi$-partioned union of $\boldsymbol{\Gamma}$ sets if for all $n<N, A \cap P_{n} \in \boldsymbol{\Gamma}_{P_{n}}$, equivalently if there exists $\left(A_{n}\right)_{n<N} \in \Gamma^{N}$ such that $A=\bigcup_{n<N} P_{n} \cap A_{n}$. Moreover given any class $\Pi$ :

- if each $P_{n}$ is in $\boldsymbol{\Pi}$ then $A$ is said to be a $\boldsymbol{\Pi}$-partitioned union of $\boldsymbol{\Gamma}$ sets.

- if $N=2$ and $\pi=\left(P_{0}, P_{1}\right) \in \mathbf{\Pi} \times \check{\boldsymbol{\Pi}}$ then $\pi$ is said to be a basic $(\boldsymbol{\Pi}, \check{\boldsymbol{\Pi}})$ partition and $A$ a basic $(\boldsymbol{\Pi}, \check{\boldsymbol{\Pi}})$-partitioned union of $\boldsymbol{\Gamma}$ sets.

Definition 2.3. Let $1 \leq \xi<\omega_{1}$. A class $\boldsymbol{\Gamma}$ is said to be of level $\geq \xi$ if $\boldsymbol{\Gamma}$ is closed under $\boldsymbol{\Delta}_{\xi}^{0}$-partitioned unions, and of level $\xi$ if moreover $\boldsymbol{\Gamma}$ is not of level $\geq \xi+1$. 
The reader can find in [6] other various formulations of the notion of level. Notice that the complement of a $\boldsymbol{\Delta}_{\xi}^{0}$-partitioned union of $\boldsymbol{\Gamma}$ sets being itself a $\boldsymbol{\Delta}_{\xi}^{0}$-partitioned union of $\check{\boldsymbol{\Gamma}}$ sets, the classes $\boldsymbol{\Gamma}$ and $\check{\boldsymbol{\Gamma}}$ are of the same level. In fact the notion of level is significant only for non self-dual classes $(\check{\boldsymbol{\Gamma}} \neq \boldsymbol{\Gamma})$. We recall that the Wadge class $\boldsymbol{\Gamma}$ generated by the set $A$ is self-dual if and only if any point in $\omega^{\omega}$ admits a $\boldsymbol{\Delta}_{1}^{0}$ neighbourhood $V$ such that the set $V \cap A$ is of class $<\boldsymbol{\Gamma}$.

Proposition 2.4. Any self-dual Wadge class is of level 1.

Proof. Suppose that $\boldsymbol{\Gamma}$ is a self-dual Wadge class of level $\geq 2$, and let $A \subset \omega^{\omega}$ be a generating set for $\boldsymbol{\Gamma}$. For all $n \leq \omega$ let $\overline{0}^{n}$ denote the sequence of length $n$ with constant value 0 , and for $n<\omega$ let $A_{n}=\left\{\overline{0}^{n} \frown \alpha: \alpha \in A\right\}$. Then the set $B=\left\{\overline{0}^{\omega}\right\} \cup \bigcup_{n \in \omega} A_{n}$ is clearly a $\boldsymbol{\Delta}_{2}^{0}$-partitioned union of $\boldsymbol{\Gamma}$ sets, hence $B \in \boldsymbol{\Gamma}$ since $\boldsymbol{\Gamma}$ is of level $\geq 2$, and since $B \cap\left(\overline{0}^{n} \times \omega^{\omega}\right)=A_{n} \approx A$ then $B$ is a generating set for $\boldsymbol{\Gamma}$. Then since $\boldsymbol{\Gamma}$ is self-dual any point $x \in \omega^{\omega}$ admits a $\Delta_{1}^{0}$ neighbourhood $V$ such that the set $V \cap B$ is of class $<\boldsymbol{\Gamma}$, but this is clearly not the case for $x=\overline{0}^{\omega}$.

Proposition 2.5. For any Wadge class $\boldsymbol{\Gamma}$ and any set $A \subset \omega^{\omega}$ let:

$$
\operatorname{ker}^{(\boldsymbol{\Gamma})}(A)=\left\{x \in \omega^{\omega}: \forall V \boldsymbol{\Delta}_{1}^{0} \text { neighbourhood of } x, V \cap A \notin \check{\boldsymbol{\Gamma}}\right\}
$$

and let $W=\omega^{\omega} \backslash \operatorname{ker}^{(\boldsymbol{\Gamma})}(A)$. Then:

a) $\operatorname{ker}^{(\boldsymbol{\Gamma})}(A)$ is a closed subset of $\omega^{\omega}$,

b) $A \cap W \in \check{\boldsymbol{\Gamma}}_{W}$, hence if $A \in \boldsymbol{\Gamma}$ then $A \cap W \in \Delta\left(\boldsymbol{\Gamma}_{W}\right)$,

c) $\operatorname{ker}^{(\boldsymbol{\Gamma})}(A)=\emptyset$ if and only if $A \in \check{\boldsymbol{\Gamma}}$,

d) if $A \in \boldsymbol{\Gamma} \backslash \check{\boldsymbol{\Gamma}}$ then for any $V \in \boldsymbol{\Delta}_{1}^{0}$ such that $V \cap \operatorname{ker}^{(\boldsymbol{\Gamma})}(A) \neq \emptyset, V \cap A \in \boldsymbol{\Gamma} \backslash \check{\boldsymbol{\Gamma}}$.

Proof. By the very definition of $\operatorname{ker}^{(\boldsymbol{\Gamma})}(A)$ it is clear that $\operatorname{ker}^{(\boldsymbol{\Gamma})}(A)$ is a closed subset of $\omega^{\omega}$ and we can find a $\boldsymbol{\Delta}_{1}^{0}$-covering $\left(V_{n}\right)_{n \in \omega}$ of $W$ such that for all $n$, $V_{n} \cap A \in \check{\boldsymbol{\Gamma}}_{V_{n}}$, hence by Proposition 2.1, $A \cap W \in \check{\boldsymbol{\Gamma}}_{W}$. This proves a) and b). For c) the implication from left to right is obvious and the converse follows from b). Finally for d) observe that if $A \in \boldsymbol{\Gamma}$ then $V \cap A \in \boldsymbol{\Gamma}$ and if moreover $V \cap \operatorname{ker}(A) \neq \emptyset$ then it follows from the definition of $\operatorname{ker}^{(\boldsymbol{\Gamma})}(A)$ that $V \cap A \notin \check{\boldsymbol{\Gamma}}$.

Proposition 2.6. Let $\boldsymbol{\Gamma}$ be a non self-dual Wadge class, let $A \in \boldsymbol{\Gamma} \backslash \check{\boldsymbol{\Gamma}}$ and set $K=\operatorname{ker}^{(\boldsymbol{\Gamma})}(A)$. Then the following are equivalent:

(i) $\boldsymbol{\Gamma}$ is of level $\geq 2$.

(ii) $\boldsymbol{\Gamma}$ is closed under basic $\left(\mathbf{\Pi}_{1}^{0}, \boldsymbol{\Sigma}_{1}^{0}\right)$-partitioned unions.

(iii) For all $V \in \boldsymbol{\Delta}_{1}^{0}$ if $V \cap K \neq \emptyset$ then $V \cap K \cap A \notin \check{\boldsymbol{\Gamma}}_{K}$.

Proof. Observe first that all three conditions are symmetrical, in the sense that whenever one of these conditions is satisfied by the class $\boldsymbol{\Gamma}$ then it is automatically satisfied by the dual class $\check{\boldsymbol{\Gamma}}$.

(i) $\Rightarrow$ (ii) is obvvious.

(ii) $\Rightarrow$ (iii): Note that since $\boldsymbol{\Gamma}$ is non trivial then for any basic $\left(\boldsymbol{\Pi}_{1}^{0}, \boldsymbol{\Sigma}_{1}^{0}\right)$ partition $\pi=(P, Q)$ and any set $A \in \boldsymbol{\Gamma}$ the set $P \cap A=(P \cap A) \cup(Q \cap \emptyset)$ is 
a $\pi$-partitioned union of $\boldsymbol{\Gamma}$ sets. It follows then from (ii) that $P \cap A \in \boldsymbol{\Gamma}$, and similarly $Q \cap A \in \boldsymbol{\Gamma}$. So $\boldsymbol{\Gamma}$ is closed under intersection with closed sets and with open sets; and the same holds for $\check{\Gamma}$.

Suppose now that (iii) fails and fix a $\boldsymbol{\Delta}_{1}^{0}$ set $V$ such that $V \cap K \neq \emptyset$ and $V \cap K \cap A \in \check{\boldsymbol{\Gamma}}_{V \cap K}$ then we can find $B \in \check{\boldsymbol{\Gamma}}$ such that $V \cap K \cap A=(V \cap K) \cap B$, and since $K \cap B \in \check{\Gamma}$ then $V \cap K \cap A=V \cap(K \cap B) \in \check{\Gamma}$. On the other hand by Proposition 2.5 a) we have that $A \backslash K \in \check{\boldsymbol{\Gamma}}_{\omega^{\omega} \backslash K}$ and we can find $B^{\prime} \in \check{\boldsymbol{\Gamma}}$ such that $A \backslash K=B^{\prime} \backslash K$

Then setting $P=V \cap K$ and $Q=V \backslash K, \pi=(P, Q)$ is a basic $\left(\boldsymbol{\Pi}_{1}^{0}, \boldsymbol{\Sigma}_{1}^{0}\right)$ partition of $V \approx \omega^{\omega}$ with $P \cap A=V \cap K \cap A \in \check{\Gamma}$ and $Q \cap A=Q \cap B^{\prime} \in \check{\Gamma}_{Q}$. Hence $V \cap A=(P \cap A) \cup\left(Q \cap B^{\prime}\right)$ is a $\pi$-partitioned union of $\check{\Gamma}$ sets, and it follows then from (ii) applied to $\check{\Gamma}_{V} \approx \check{\Gamma}$ that $V \cap A \in \check{\boldsymbol{\Gamma}}$ which is a contradiction since $V \cap \operatorname{ker}^{(\boldsymbol{\Gamma})}(A) \neq \emptyset$.

(iii) $\Rightarrow$ (i): Suppose that $\boldsymbol{\Gamma}$, equivalently $\check{\boldsymbol{\Gamma}}$, is of level 1 . Then we can find a $\boldsymbol{\Pi}_{1}^{0}$ partition $\left(F_{n}\right)_{n \in \omega}$ of $\omega^{\omega}$ and a set $B \notin \check{\boldsymbol{\Gamma}}$ such that for all $n \in \omega, F_{n} \cap B=F_{n} \cap B_{n}$ for some $B_{n} \in \check{\boldsymbol{\Gamma}}$. So $B=\bigcup_{n \in \omega} F_{n} \cap B_{n}$ is Borel and since $B \notin \check{\Gamma}$ then $B$ reduces any set in $\boldsymbol{\Gamma}$. In particular since $A \in \boldsymbol{\Gamma}$ then we can find a continuous function $\varphi: \omega^{\omega} \rightarrow \omega^{\omega}$ such that $A=\varphi^{-1}(B)$. Then for all $n \in \omega, F_{n}^{\prime}:=\varphi^{-1}\left(F_{n}\right) \in \Pi_{1}^{0}$, $A_{n}:=\varphi^{-1}\left(B_{n}\right) \in \check{\boldsymbol{\Gamma}}$ and $F_{n}^{\prime} \cap A=F_{n}^{\prime} \cap A_{n}$. Since $A \notin \check{\boldsymbol{\Gamma}}$ then $K=\operatorname{ker}(A)$ is a non empty Polish space, and applying Baire Theorem we can find a $\boldsymbol{\Delta}_{1}^{0}$ set $V$ and some $n$ such that $\emptyset \neq V \cap K \subset F_{n}^{\prime}$ hence by (iii), $V \cap K \cap A \notin \check{\boldsymbol{\Gamma}}_{V \cap K}$. But $V \cap K \cap A=V \cap K \cap F_{n}^{\prime} \cap A=V \cap K \cap A_{n} \in \check{\boldsymbol{\Gamma}}_{V \cap K}$ which is a contradiction.

Corollary 2.7. If $\boldsymbol{\Gamma}$ is a non self-dual Wadge class of level 1 then for any set $A \in \boldsymbol{\Gamma}$ there exists a basic $\left(\boldsymbol{\Pi}_{1}^{0}, \boldsymbol{\Sigma}_{1}^{0}\right)$-partition $(P, Q)$ such that $A \cap P \in \check{\boldsymbol{\Gamma}}_{P}$ and $A \cap Q \in \check{\boldsymbol{\Gamma}}_{Q}$.

Proof. Applying Proposition 2.6 (iii) to some fixed set $B \in \boldsymbol{\Gamma} \backslash \check{\boldsymbol{\Gamma}}$, we can find a $\boldsymbol{\Delta}_{1}^{0}$ set $V$ such that $P_{0}=V \cap \operatorname{ker}^{(\boldsymbol{\Gamma})}(B)$ is non empty and $P_{0} \cap B \in \check{\boldsymbol{\Gamma}}_{P_{0}}$. Then setting $Q_{0}=V \backslash \operatorname{ker}(B)$ we have by Proposition 2.5 a) that $Q_{0} \cap B \in \check{\boldsymbol{\Gamma}}_{Q_{0}}$. This proves Corollary 2.7 for $A=V \cap B$.

Since $V \cap \operatorname{ker}^{(\boldsymbol{\Gamma})}(B) \neq \emptyset$ then $V \cap B \in \boldsymbol{\Gamma}_{V} \backslash \check{\boldsymbol{\Gamma}}_{V}$ and since $V \approx \omega^{\omega}$ then the set $B \cap V$ as a subspace of $V$ is $\boldsymbol{\Gamma}$-complete. Then for any set $A \in \boldsymbol{\Gamma}$ we can find a continuous function $\varphi: \omega^{\omega} \rightarrow V$ such that $A=\varphi^{-1}(B)$; and setting $P=\varphi^{-1}\left(P_{0}\right)$, and $Q=\varphi^{-1}\left(Q_{0}\right)$ it is clear that the partition $(P, Q)$ satisfies the conclusion of Corollary 2.7.

\section{A variation of the game operator D}

For any set $E \subset \omega^{\omega}$ we denote by $\mathbf{G}_{E}$ the standard free game on $\omega$ in which $E$ is the payoff set for Player I. If $X \subset \omega^{\omega}$ and $A \subset \omega^{\omega} \times \omega^{\omega}$ let:

$$
\partial^{X} A=\left\{x \in X: \text { Player I wins the game } \mathbf{G}_{A(x)}\right\}
$$


and if $\Gamma$ is a given class we denote by $\partial^{X} \Gamma$ the class of all subsets of $X$ of the form $\partial^{X} A$ with $A \subset \omega^{\omega} \times \omega^{\omega}$ in $\Gamma$. When $X=\omega^{\omega}$ we shall use the standard notation and write $\partial$ instead of $\partial^{\omega^{\omega}}$.

For all basic results on the operator $\partial$ we refer the reader to [7]. Let us only mention that for any class $\Gamma$, since $\Gamma$ is closed under recursive substitutions, $\exists^{\omega} \partial \Gamma \subset \partial \Gamma$ and $\forall^{\omega} \partial \Gamma \subset \partial \Gamma$. In particular if $\Gamma$ is closed under continuous substitutions then $\partial \Gamma$ is closed under countable unions and intersections.

Let $\mathcal{T}$ denote the set of all trees on $\omega$ with no terminal nodes, that we identify to a $\Pi_{2}^{0}$ subset of the compact space $2^{\omega^{<\omega}}$. Given any $T \in \mathcal{T}$ we denote by $\mathbf{G}^{T}$ the game on $\omega$ with rules $T$, meaning that any finite run in $\mathbf{G}^{T}$ has to be an element of $T$. Hence the set of all infinite runs in $\mathbf{G}^{T}$ is just the set $\lceil T\rceil$ of all infinite branches of $T$. Then given any set $E \subset\lceil T\rceil$ we denote by $\mathbf{G}_{E}^{T}$ the game with rules $T$ with $E$ as payoff set for Player I.

Also given any mapping $\Phi: X \subset \omega^{\omega} \rightarrow \mathcal{T}$ and any set $A \subset \omega^{\omega} \times \omega^{\omega}$ we define:

$$
\partial_{A}^{\Phi}:=\left\{x \in X: \text { Player I wins the game } \mathbf{G}_{A(x)}^{\Phi(x)}\right\}
$$

and given any class $\Gamma$ we denote by $\partial^{\Phi} \Gamma$ the class of all subsets of $X$ of the form $\partial_{A}^{\Phi}$ with $A \subset \omega^{\omega} \times \omega^{\omega}$ in $\Gamma$. Note that if $\Phi_{X}$ denotes the constant mapping $x \mapsto \omega^{<\omega}$ from $X$ to $\mathcal{T}$ then $\partial^{\Phi_{X}}$ is just $\partial^{X}$.

Proposition 3.1. Let $\Phi: X \rightarrow \mathcal{T}$ and $\hat{\Phi}: X \rightarrow X \times \mathcal{T}$ the mapping defined by $\hat{\Phi}(x)=(x, \Phi(x))$. Then for any set $A \subset \omega^{\omega} \times \omega^{\omega}$ in some class $\Gamma$ there exists a set $\hat{A} \subset\left(\omega^{\omega} \times \mathcal{T}\right) \times \omega^{\omega}$ in $\Gamma$ such that $\partial^{\Phi} A=\hat{\Phi}^{-1}(\partial \hat{A})$.

Proof. Let $\Psi: \mathcal{T} \times \omega^{<\omega} \rightarrow \omega^{<\omega}$ defined by $\Psi(T, \emptyset)=\emptyset$ and for all $s \in \omega^{<\omega}$, if $\Psi(T, s)=t$ then for all $m \in \omega, \Psi\left(T, s^{\frown} m\right)=t \frown n$ with:

$$
n= \begin{cases}\max \{k \leq m: t \frown k \in T\} & \text { if } \exists k \leq m: t^{\frown k \in T} \\ \min \{k: t \frown k \in T\} & \text { if not }\end{cases}
$$

which is well defined since $T$ has no terminal node.

Then for all $T \in \mathcal{T}, \Psi(T, \cdot)$ induces a tree homomorphism from $\omega^{\omega}$ onto $T$, and one easily checks by induction that for all $s \in T, \Psi(T, s)=s$.

Then the function $\psi: \mathcal{T} \times \omega^{\omega} \rightarrow \omega^{\omega}$ defined by setting for all $\alpha \in \omega^{\omega}$ :

$$
\psi(T, \alpha):=\bigcup_{n \in \omega} \Psi\left(T, \alpha_{\mid n}\right)
$$

is clearly recursive. Moreover for all $T \in \mathcal{T}$, the function $\psi(T, \cdot)$ is a Lipschitz retraction from $\omega^{\omega}$ onto $\lceil T\rceil$; in particular for all $E \subset \omega^{\omega}, \psi(\{T\} \times E)=E \cap\lceil T\rceil$.

If $A \subset \omega^{\omega} \times \omega^{\omega}$ in $\Gamma$ then the set

$$
\hat{A}=\left\{(x, T, \alpha) \in \omega^{\omega} \times \mathcal{T} \times \omega^{\omega}:(x, \psi(T, \alpha)) \in A\right\}
$$

is in $\Gamma$ too and for all $(x, T) \in X \times \mathcal{T}$ :

- If $\sigma$ is a winning strategy for Player I in the game $\mathbf{G}_{\hat{A}(x, T)}$ then $\sigma$ is necessarily valued in $\bigcup_{n} T \cap \omega^{2 n+1}$, so $\tau=\sigma_{\mid \bigcup_{n} T \cap \omega^{2 n}}$ is a winning strategy for Player I in the game $\mathbf{G}_{A(x)}^{T}$. 
- If $\tau$ is a winning strategy for Player I in the game $\mathbf{G}_{A(x)}^{T}$ and if for all $\sigma \in \omega^{2 n}$ we define $\sigma(s)=\tau(\Psi(T, s)) \in T \cap \omega^{2 n+1}$ then $\sigma$ is clearly a winning strategy for Player I in the game $\mathbf{G}_{A(x) \cap\lceil T\rceil}=\mathbf{G}_{\hat{A}(x, T)}$.

In particular for all $x \in X$ :

Player I wins the game $\mathbf{G}_{A(x)}^{\Phi(x)} \Longleftrightarrow$ Player I wins the game $\mathbf{G}_{\hat{A}(x, \Phi(x))}$ hence $\supset^{\Phi} A=\hat{\Phi}^{-1}(\supset \hat{A})$.

\section{The GAME OPERATOR ON WADGE ClASSES OF LEVEL $\geq 2$}

We recall the following notion from [7]:

Definition 4.1. (Moschovakis) A given class $\Sigma$ is said to have the substitution property if for any partial $\Sigma$-recursive function $f: D \subset \omega^{\omega} \rightarrow \omega^{\omega}$ and any set $B \in \Sigma$ there exists a set $A \in \Sigma$ such that $f^{-1}(B)=A \cap D$.

Let us point out that dealing with partial $\Sigma$-recursive functions rather than total ones is fundamental for most uses of this notion. Note also that if $\exists^{\omega} \Sigma \subset \Sigma$ then any partial $\check{\Sigma}$-recursive function is also $\Sigma$-recursive, hence if $\Sigma$ has the substitution property then so does $\check{\Sigma}$.

The substitution property is actually a very strong closure property not easy to verify in general, and the following stability result is one of the rare results ensuring it. In fact, though not stated explicitly by Moschovakis, this result is essentially contained in the proof of Theorem 6D. 4 in [7]. For completeness we give here a slightly modified and simpler version of Moschovakis' proof.

Theorem 4.2. Given a class $\Gamma$ let $\boldsymbol{\Gamma}=\bigcup_{\varepsilon \in \omega^{\omega}} \Gamma(\varepsilon)$ and assume $\operatorname{Det}(\boldsymbol{\Gamma})$. If $\Gamma$ is closed under basic $\left(\Pi_{1}^{0}, \Sigma_{1}^{0}\right)$-partitioned unions then $\partial \Gamma$ has the substitution property.

Proof. Let $f: D \subset \omega^{\omega} \rightarrow \omega^{\omega}$ be a partial $\partial \Gamma$-recursive function and $B \in \partial \Gamma$. We have to find a set $A \in \partial \Gamma$ such that $f^{-1}(B)=D \cap A$.

Since $B \in \partial \Gamma$ we can fix a set $\hat{B} \subset \omega^{\omega} \times \omega^{\omega}$ in $\Gamma$ such that $B=\partial \hat{B}$. Also since $f$ is $\partial \Gamma$-recursive we can fix a set $L \subset \omega^{<\omega} \times \omega^{\omega}$ in $\partial \Gamma$ such that for all $s \in \omega^{<\omega}, D \cap f^{-1}\left(N_{s}\right)=D \cap L_{s}$ where $L_{s}=L(s) \subset \omega^{\omega}$. Let

$$
M=\left\{(s, x) \in \omega^{<\omega} \times \omega^{\omega}: \exists t \in \omega^{<\omega}, N_{s} \cap N_{t}=\emptyset \text { and } x \in L_{t}\right\}
$$

so that for all $s \in \omega^{<\omega}, D \backslash f^{-1}\left(N_{s}\right)=D \cap M_{s}$ with a set $M \subset \omega^{<\omega} \times \omega^{\omega}$ in $\partial \Gamma$; so we can fix $\hat{M} \subset \omega^{<\omega} \times \omega^{\omega} \times \omega^{\omega}$ in $\Gamma$ such that $M=\partial \hat{M}$. Since we assume $\operatorname{Det}(\boldsymbol{\Gamma})$ then for all $x \in D$ :

$$
\begin{aligned}
& f(x) \notin N_{s} \quad \Longleftrightarrow \quad \text { Player I wins the game } \mathbf{G}_{\hat{M}_{s}(x)} \\
& f(x) \in N_{s} \quad \Longleftrightarrow \quad \text { Player II wins the game } \mathbf{G}_{\hat{M}_{s}(x)} .
\end{aligned}
$$

For all $x \in \omega^{\omega}$ consider the game $H_{x}$ which runs as follows:

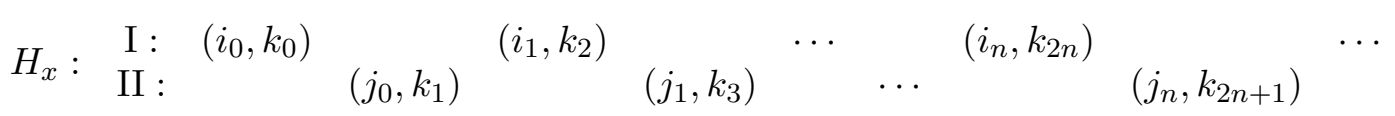


with $i_{n}, j_{n}, k_{n} \in \omega$.

We shall identify any infinite run $\rho$ in $H_{x}$ to a triple $(y, z, \alpha) \in\left(\omega^{\omega}\right)^{3}$ with:

$$
y=\left(i_{n}\right)_{n \in \omega} \quad, \quad z=\left(j_{n}\right)_{n \in \omega} \quad, \quad \alpha=\left(k_{n}\right)_{n \in \omega}
$$

and refer to $y$ as the real constructed by Player $I$ in this run. We also set for all $p \in \omega:$

$$
\alpha^{(p)}=\left(k_{p+n}\right)_{n \in \omega} \in \omega^{\omega} .
$$

Then Player I wins the run $\rho=(y, z, \alpha)$ if:

$\left(\forall n \in \omega, y_{\mid n}=z_{\mid n}\right.$ and $\left.(y, \alpha) \in \hat{B}\right)$ or $\left(\exists n \in \omega, y_{\mid n} \neq z_{\mid n}\right.$ and $\left.\left(x, \alpha^{(2 n+2)}\right) \in \hat{M}_{s_{n}}\right)$.

Lemma 4.3. The set $A=\left\{x \in \omega^{\omega}\right.$ : Player I wins the game $\left.H_{x}\right\}$ is in $\partial \Gamma$.

Proof. We shall define a set $\hat{A} \subset \Omega:=\omega^{\omega} \times\left(\omega^{\omega} \times \omega^{\omega} \times \omega^{\omega}\right)$ such that for all $x \in \omega^{\omega}$, Player I wins the game $H_{x}$ if and only if Player I wins the game $\mathbf{G}_{\hat{A}(x)}$. For this let:

$$
\begin{gathered}
P=\left\{(x, y, z, \alpha) \in \Omega: \forall n \in \omega, y_{\mid n}=z_{\mid n}\right\} \quad ; \quad Q=\Omega \backslash P \\
\hat{B}_{\emptyset}=\{(x, y, z, \alpha) \in \Omega:(y, \alpha) \in \hat{B}\}
\end{gathered}
$$

and for all $s \in \omega^{n} \backslash\{\emptyset\}$ :

$$
\begin{gathered}
V_{s}=\left\{(x, y, z, \alpha) \in \Omega: \forall m<n \in \omega, y_{\mid m}=z_{\mid m} \text { and } y_{\mid n} \neq z_{\mid n}=s\right\} \\
\hat{B}_{s}=\left\{(x, y, z, \alpha) \in \Omega:\left(x, \alpha^{(2|s|+2)}\right) \in \hat{M}_{s}\right\} .
\end{gathered}
$$

and

$$
\hat{A}=\left(P \cap \hat{A}_{\emptyset}\right) \cup \bigcup_{\emptyset \neq s \in \omega<\omega} V_{s} \cap \hat{A}_{s}
$$

Then $\hat{A} \cap P=\hat{A}_{\emptyset} \cap P$ with $\hat{A}_{\emptyset} \in \Gamma$ hence $\hat{A} \cap P \in \Gamma_{P}$; and $\hat{A} \cap Q=\bigcup_{\emptyset \neq s \in \omega<\omega} V_{s} \cap \hat{A}_{s}$ is locally in $\Gamma_{Q}$ hence in $\Gamma_{Q}$. This proves that $\hat{A}$ is a basic $\left(\Pi_{1}^{0}, \Sigma_{1}^{0}\right)$-partitioned union of $\Gamma$ sets hence $\hat{A} \in \Gamma$; and clearly for all $x$, Player I wins the run $(y, z, \alpha)$ in $H_{x}$ if and only $(x, y, z, \alpha) \in \hat{A}$.

Lemma 4.4. For all $x \in D$,

a) if Player I wins the game $\mathbf{G}_{\hat{B}(f(x))}$ then Player I wins the game $H_{x}$.

b) if Player II wins the game $\mathbf{G}_{\hat{B}(f(x))}$ then Player II wins the game $H_{x}$.

Proof. Fix $x \in D$ and set $z=f(x)$. We shall describe in each case a strategy for the concerned Player, and it will follow readily from the win conditions of the game that these strategies are winning.

a) Let $\sigma$ be a winning strategy for Player $I$ in the game $\mathbf{G}_{\hat{B}(x)}$. Then in any run in $H_{x}$ Player I plays $i_{n}=z(n)$ for all $n$, so that in any infinite run $z=y$ is the real constructed by Player I in the run. Then as long as $s_{n}=y_{\mid n}$ Player I plays $k_{2 n}$ following $\sigma$, and at the first Player II's move for which $s_{p} \neq y_{\mid p}$, in which case $y=f(x) \notin N_{s_{p}}$, Player I starts playing $k_{2 p+2+2 n}$ for $n \geq 0$ following a winning strategy in the game $\mathbf{G}_{\hat{M}_{s_{p}}(x)}$. 
b) Let $\tau$ be a winning strategy for Player II in the game $\mathbf{G}_{\hat{B}(x)}$. Then in any run in $H_{x}$ Player II plays $s_{n}=z_{\mid n}$ for all $n$. If $y$ is the real constructed by Player I in the run then as long as $s_{n}=z_{n}=y_{\mid n}$ Player II plays $k_{2 n+1}$ following $\tau$, and at the first Player I's move for which $s_{p} \neq z_{\mid p}$, in which case $y \neq f(x)$ and $f(x) \in N_{s_{p}}$, Player II starts playing $k_{2 p+1+2 n}$ for $n \geq 0$ following a winning strategy in the game $\mathbf{G}_{\hat{M}_{s p}(x)}$.

Since $\operatorname{Det}(\boldsymbol{\Gamma})$ holds then the game $\mathbf{G}_{\hat{B}(f(x))}$ is determined, hence by Lemma 4.4 the game $H_{x}$ is determined too and for all $x \in D$ we have:

$$
\begin{aligned}
f(x) \in B & \Longleftrightarrow \text { Player I wins the game } \mathbf{G}_{\hat{B}(f(x))} \\
& \Longleftrightarrow \text { Player I wins the game } H_{x} \\
& \Longleftrightarrow x \in A
\end{aligned}
$$

Hence $f^{-1}(B)=D \cap A$ and by Lemma 4.3 the set $A$ is in $\partial \Gamma$, which finishes the proof of Theorem 4.2.

Corollary 4.5. If $\boldsymbol{\Gamma}$ is a Wadge class of level $\geq 2$ then $\partial \boldsymbol{\Gamma}$ has the substitution property.

The converse of Corollary 4.5 is not true since $\boldsymbol{\Sigma}_{1}^{1}=\partial \boldsymbol{\Pi}_{1}^{0}$ and $\boldsymbol{\Pi}_{1}^{1}=\partial \boldsymbol{\Sigma}_{1}^{0}$ do have the substitution property while $\boldsymbol{\Pi}_{1}^{0}$ and $\boldsymbol{\Sigma}_{1}^{0}$ are of level 1 . However as we shall see these are the only possible counter-examples. The proof relies on the following general determinacy result for which we need first to fix some notation.

Notation 4.6. For all $u \in \omega^{<\omega}$ let:

$$
S_{u}=\left\{s \in \omega^{<\omega}: s \supset u\right\} \quad \text { and } \quad \mathrm{J}_{u}=\left\{\begin{array}{cc}
\mathrm{I} & \text { if }|u| \text { is even } \\
\mathrm{II} & \text { if }|u| \text { is odd }
\end{array}\right.
$$

So $S_{u} \in \mathcal{T}$ and for any set $E \subset \omega^{\omega}$ we can consider the game $\mathbf{G}_{E}^{S_{u}}$. Then a run in $\mathbf{G}_{E}^{S_{u}}$ is just a run in the standard free game $\mathbf{G}_{E}$ which extends $u$, and the moves of Player $\mathrm{J}_{u}$ in the game $\mathbf{G}_{E}^{S_{u}}$ are in one-to-one correspondence with the moves of Player I in the standard free game $\mathbf{G}_{E}$.

Proposition 4.7. Let $B, W \subset \omega^{\omega}$ with $W \in \boldsymbol{\Sigma}_{1}^{0}$. Then Player I wins the game $\mathbf{G}_{B \cup W}$ if and only if one of the following two conditions holds:

i) Player I wins the game $\mathbf{G}_{W}$,

ii) Player II wins the game $\mathbf{G}_{W}$ and Player I wins the game $\mathbf{G}_{B}^{T_{W}}$, where

$$
T_{W}=\left\{t \in \omega^{<\omega}: \forall u \subset t \text {, Player } J_{u} \text { has no winning strategy in } \mathbf{G}_{W}^{S_{u}}\right\} \text {. }
$$

Proof. a) Set for simplicity $T=T_{W}$ and suppose that (i) or (ii) holds:

If Player I wins the game $\mathbf{G}_{W}$ then he obviously also wins the game $G_{B \cup W}$ by following the same strategy.

So suppose that Player II wins the game $\mathbf{G}_{W}$. Then by the proof of the GaleStewart theorem, $T=T_{W}$ is a winning quasi-strategy for Player II in the game $\mathbf{G}_{W}$, hence $T \in \mathcal{T}$ and $\lceil T\rceil \cap W=\emptyset$. Moreover if $u \in \omega^{<\omega} \backslash T$ is of minimal length (i.e. $u \notin T$ and $u^{*} \in T$ ) then by definition of $T$ Player $\mathrm{J}_{u}$ has a winning strategy, say $\sigma_{u}$, in $\mathbf{G}_{W}^{S_{u}}$. Note that since $T \in \mathcal{T}$ then the game $G_{B \backslash W}^{T}$ is well defined, 
and if Player I wins this game with some strategy $\sigma$, then Player I has a winning strategy $\tau$ in $\mathbf{G}_{B \cup W}$ by following $\sigma$ as long as the run is in $T$ and if $u \in \omega^{n}$ is a run of minimal length, which is compatible with $\sigma$ but not in $T$, then from step $n$ on, Player I (who corresponds to Player $\mathrm{J}_{u}$ in $\mathbf{G}^{S_{u}}$ ) follows the strategy $\sigma_{u}$. Note that such a run $u$ might be of even length (if Player I gets out of $T$ by the requirements of the strategy $\sigma$ ) or of odd length (if Player II decides freely to get out of $T$ ). Then for any infinite run $\alpha$ compatible with $\tau$ :

- either $\alpha \in\lceil T\rceil$ and then $\alpha$ can be viewed as an infinite run in $\mathbf{G}_{B}^{T}$ compatible with $\sigma$, hence $\alpha \in B$

- or else for some minimal $n, \alpha_{\mid n}=u$ is compatible with $\sigma$ but $u \notin T$, and then $\alpha$ is compatible with the strategy $\sigma_{u}$, hence $\alpha \in W$.

This proves that the strategy $\tau$ is winning for Player I in the game $\mathbf{G}_{B \cup W}$.

b) Conversely suppose that Player I wins the game $\mathbf{G}_{B \cup W}$ but does not win the game $\mathbf{G}_{W}$ then Player II wins the game $\mathbf{G}_{B}^{T}$. More precisely if $\sigma$ is any winning strategy for Player I in the game $\mathbf{G}_{B \cup W}$ then $\sigma$ is also a winning strategy for Player $\mathrm{I}$ in the game $\mathbf{G}_{B}^{T}$. Indeed since $T$ is a quasi-strategy for Player II in the game $\mathbf{G}_{W}$ then for all $s \in T \cap \omega^{2 n}$ and all $k \in \omega, s \frown k \in T$. Thus in any infinite run $\alpha \in \omega^{\omega}$, as long as Player II stays in $T$, Player I is forced to stay in $T$ too, and then $\alpha \in\lceil T\rceil \subset \omega^{\omega} \backslash V$; and if moreover $\alpha$ is compatible with $\sigma$ then $\alpha \in(B \cup W) \cap\lceil T\rceil=B \cap\lceil T\rceil$ since $W \cap\lceil T\rceil=\emptyset$. This proves that the strategy $\sigma$ is winning in the game $\mathbf{G}_{B}^{T}$.

Given two classes $\Sigma, \Lambda$ and a function $f$ we shall write informally $f^{-1}(\Sigma) \subset \Lambda$ to mean that the inverse image by $f$ of any set in $\Sigma$ is in $\Lambda$.

Definition 4.8. We shall say that a given class $\Sigma$ has the weak substitution property if $f^{-1}(\Sigma) \subset \Sigma$ for any total function $f: \omega^{\omega} \rightarrow \omega^{\omega}$, equivalently for any partial function $f: D \subset \omega^{\omega} \rightarrow \omega^{\omega}$ with domain $D$ in $\Delta(\Sigma)$.

Note that if $\boldsymbol{\Lambda}$ is a class closed under continuous substitutions and countable unions, in particular if $\boldsymbol{\Lambda}=\partial \boldsymbol{\Gamma}$ for some Wadge class $\boldsymbol{\Gamma}$, then for a total function $f$ the notion of $\boldsymbol{\Lambda}$-recursivity coincides with the notion of $\boldsymbol{\Lambda}$-measurability, that is $f^{-1}\left(\boldsymbol{\Sigma}_{1}^{0}\right) \subset \boldsymbol{\Lambda}$.

We can now sate the following converse to Theorem 4.2.

Theorem 4.9. Let $\boldsymbol{\Gamma}$ be a non self-dual Wadge class containing the class $\boldsymbol{\Pi}_{1}^{0} \cup \boldsymbol{\Sigma}_{1}^{0}$. If $\supset \boldsymbol{\Gamma}$ has the weak substitution property then $\boldsymbol{\Gamma}$ is of level $\geq 2$.

Proof. Fix a recursive isomorphism $\imath:\left(\omega^{\omega}\right)^{2} \rightarrow \omega^{\omega}$ and let $\jmath:\left(\omega^{\omega}\right)^{3} \rightarrow\left(\omega^{\omega}\right)^{2}$ be defined by $\jmath(x, y, z)=(\imath(x, y), z)$. If $U \subset \omega^{\omega} \times\left(\omega^{\omega} \times \omega^{\omega}\right)$ is universal for the $\boldsymbol{\Gamma}$ subsets of $\omega^{\omega} \times \omega^{\omega}$ then $A=\jmath(U) \subset \omega^{\omega} \times \omega^{\omega}$ is in $\boldsymbol{\Gamma}$ so $\supset A \subset \omega^{\omega}$ is in $\partial \boldsymbol{\Gamma}$, and one easily checks that $\imath^{-1}(\partial A) \subset \omega^{\omega} \times \omega^{\omega}$ is universal for the $\partial \boldsymbol{\Gamma}$ subsets of $\omega^{\omega}$. Hence $A \in \boldsymbol{\Gamma}$ and $\partial A \in \partial \boldsymbol{\Gamma} \backslash \partial \check{\boldsymbol{\Gamma}}$.

Suppose now that $\boldsymbol{\Gamma}$ is of level 1. Since $A \in \boldsymbol{\Gamma}$ then by Corollary 2.7 we can find a basic $\left(\boldsymbol{\Pi}_{1}^{0}, \boldsymbol{\Sigma}_{1}^{0}\right)$-partition $(F, W)$ of $\omega^{\omega}$ such that $A \cap F \in \check{\boldsymbol{\Gamma}}_{F}$ and $A \cap W \in \check{\boldsymbol{\Gamma}}_{W}$. Hence we can fix $B \in \check{\boldsymbol{\Gamma}}$ such that $A \cap F=B \cap F$, and we can also fix a $\boldsymbol{\Delta}_{1}^{0}$ 
partition $\left(V_{n}\right)_{n \in \omega}$ of $W$ such that for all $n, V_{n}=N_{s_{n}} \times N_{t_{n}}$ is a basic $\boldsymbol{\Delta}_{1}^{0}$ subset of $\omega^{\omega} \times \omega^{\omega}$, and $A_{n}:=A \cap V_{n}$ is in $\check{\boldsymbol{\Gamma}}_{V_{n}}$ hence $A_{n} \in \Delta\left(\boldsymbol{\Gamma}_{V_{n}}\right) \subset \Delta(\boldsymbol{\Gamma})$.

Then let $\Psi: \omega^{\omega} \rightarrow 2^{\omega}$ be the function whose $n^{\text {th }}$ coordinate $\Psi^{(n)}$ is the characteristic function of the set $\partial A_{n}$, so:

$$
\Psi^{(n)}(x)=1 \Longleftrightarrow \text { Player I wins the game } \mathbf{G}_{A_{n}(x)}^{S_{t_{n}}} .
$$

and let $\tilde{\Psi}: \omega^{\omega} \times \omega^{\omega} \rightarrow \omega^{\omega} \times 2^{\omega} \times \omega^{\omega}$ defined by $\tilde{\Psi}(x, \alpha)=(x, \Psi(x), \alpha)$. Since each $A_{n}$ is in $\Delta(\boldsymbol{\Gamma})$ then $\Psi$ and $\tilde{\Psi}$ are $\partial \boldsymbol{\Gamma}$-measurable.

Let $\tilde{A}, \tilde{B}, \tilde{W}$ be the subsets of $\Omega:=\omega^{\omega} \times 2^{\omega} \times \omega^{\omega}$ defined by:

$$
\begin{gathered}
\tilde{A}=\tilde{B} \cup \tilde{W} \\
\tilde{B}=\{(x, y, \alpha) \in \Omega:(x, \alpha) \in B\} \\
\tilde{W}=\left\{(x, y, \alpha) \in \Omega: \exists n \in \omega,(x, \alpha) \in V_{n} \text { and } y(n)=1\right\}
\end{gathered}
$$

and note that $\tilde{B} \in \check{\boldsymbol{\Gamma}}$ and $\tilde{W} \in \boldsymbol{\Sigma}_{1}^{0}$.

Claim 4.10. $\partial A=\tilde{\Psi}^{-1}(\partial \tilde{A})$.

Proof: Fix $x \in \omega^{\omega}$ and let $y=\Psi(x)$; we have to show that:

Player I wins the game $\mathbf{G}_{A(x)} \Longleftrightarrow$ Player I wins the game $\mathbf{G}_{\tilde{A}(x, y)}$

If Player I wins the game $\mathbf{G}_{A(x)}$ with some strategy $\sigma$ then Player I wins the game $\mathbf{G}_{\tilde{A}(x, y)}$ by following the same strategy $\sigma$. Indeed suppose that $\alpha$ is an infinite run compatible with $\sigma$; then $(x, \alpha) \in A$ and:

- either $(x, \alpha) \in F$ and then $(x, \alpha) \in A \cap F=B \cap F$ hence $(x, y, \alpha) \in \tilde{B} \subset \tilde{A}$,

- or $(x, \alpha) \in W$ and then there exists some $k \in \omega$ such that $(x, \alpha)_{\mid k}=\left(s_{n}, t_{n}\right)$ and we claim that we necessarily have $y(n)=1$. For otherwise Player II would have a winning strategy $\tau$ in the game $\mathbf{G}_{A_{n}(x)}^{S_{t_{n}}}$, and playing $\tau$ against $\sigma$ from $k$ on, would lead to an infinite run $\alpha^{\prime}$ compatible with $\sigma$ with $\left(x, \alpha^{\prime}\right) \in V_{n} \backslash A_{n} \subset W \backslash A$ which is impossible since the strategy $\sigma$ is winning in $\mathbf{G}_{A(x)}$. Hence $y(n)=1$ and so $(x, y, \alpha) \in \tilde{W} \subset \tilde{A}$.

Conversely if Player I wins the game $\mathbf{G}_{\tilde{A}(x, y)}$ with some strategy $\tilde{\sigma}$ then he has a winning strategy $\sigma$ in the game $\mathbf{G}_{A(x)}$ by following $\tilde{\sigma}$ as long as the run is of the form $t_{n}$ with $x \in N_{s_{n}}$ and $y(n)=0$; and at the first step at which $y(n)=1$ Player $\mathrm{I}$ is then in a position to win the game $\mathbf{G}_{A_{n}(x)}^{S_{t_{n}}}$, he then follows from $k$ on a winning strategy in this latter game, which constructs an infinite run in $A_{n}(x) \subset A(x)$. This proves that the strategy $\sigma$ is winning in the game $\mathbf{G}_{A(x)}$. $\diamond$

Claim 4.11. $\partial \tilde{A} \in \partial \check{\Gamma}$.

Proof: Let $Z=\omega^{\omega} \times 2^{\omega} \backslash \partial \tilde{W}$ and consider $\Phi: Z \rightarrow \mathcal{T}$ where for all $z \in Z$,

$$
\Phi(z)=\left\{t \in \omega^{<\omega}: \forall u \subset t \text {, Player } \mathrm{J}_{u} \text { has no winning strategy in } \mathbf{G}_{\tilde{W}(z)}^{S_{u}}\right\} .
$$

is the Gale-Stewart quasi strategy for Player II in the game $\mathbf{G}_{\tilde{W}(z)}$. Since $\tilde{W} \in \boldsymbol{\Sigma}_{1}^{0}$ then $\partial \tilde{W} \in \boldsymbol{\Pi}_{1}^{1} \subset \partial \check{\boldsymbol{\Gamma}}$, and since $\boldsymbol{\Sigma}_{1}^{1} \subset \partial \boldsymbol{\Gamma}$ then $\partial \tilde{W} \in \Delta(\partial \boldsymbol{\Gamma})$. Moreover since 
the relation $t \in \Phi(z)$ is $\boldsymbol{\Sigma}_{1}^{1} \subset \Delta(\partial \boldsymbol{\Gamma})$ then the function $\Phi$ is $\Delta(\partial \boldsymbol{\Gamma})$-measurable with domain in $\Delta(\partial \boldsymbol{\Gamma})$.

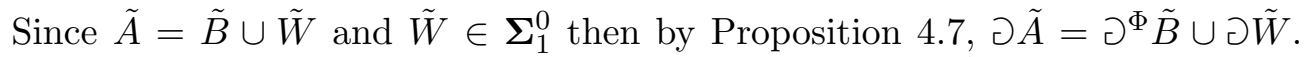
And since $\tilde{B} \in \check{\Gamma}$ then by Proposition 3.1 there exists a set $\hat{B} \subset Z \times \mathcal{T}$ in $\check{\Gamma}$ such that $\supset^{\Phi} \tilde{B}=\hat{\Phi}^{-1}(\supset \hat{B})$ where $\hat{\Phi}: Z \rightarrow Z \times \mathcal{T}$ is defined by $\hat{\Phi}(z)=(z, \Phi(z))$. So $\hat{\Phi}$ is $\Delta(\partial \boldsymbol{\Gamma})$-measurable with domain in $\Delta(\partial \boldsymbol{\Gamma})$ and it follows then from the weak substitution property of $\circlearrowright \check{\boldsymbol{\Gamma}}$ that $\partial^{\Phi} \tilde{B}=\hat{\Phi}^{-1}(\partial \hat{B}) \in \partial \check{\boldsymbol{\Gamma}}$. Then since $\circlearrowright \check{\boldsymbol{\Gamma}}$ is closed under countable unions, $\partial \tilde{A} \in \partial \check{\Gamma}$.

Finally since $\Psi$ and $\tilde{\Psi}$ are total $\partial \boldsymbol{\Gamma}$-measurable functions then it follows again from the weak substitution property of $\partial \check{\boldsymbol{\Gamma}}$ that $\partial A=\tilde{\Psi}^{-1}(\partial \tilde{A}) \in \partial \check{\boldsymbol{\Gamma}}$, which contradicts the choice of the set $A$ and finishes the proof of Theorem 4.9.

Corollary 4.12. Let $\boldsymbol{\Gamma}$ be a non self-dual Wadge class and let $\Gamma \supset \Pi_{1}^{0} \cup \Sigma_{1}^{0}$ be a class such that $\boldsymbol{\Gamma}=\bigcup_{\varepsilon \in \omega^{\omega}} \Gamma(\varepsilon)$. If $\partial \Gamma$ has the substitution property then $\boldsymbol{\Gamma}$ is of level $\geq 2$.

Proof. Since $\Gamma \supset \Pi_{1}^{0} \cup \Sigma_{1}^{0}$ then $\boldsymbol{\Gamma} \supset \boldsymbol{\Pi}_{1}^{0} \cup \boldsymbol{\Sigma}_{1}^{0}$. To see that $\boldsymbol{\Gamma}$ has the substitution property observe that given any partial $\Gamma(\varepsilon)$-recursive function $f: D \subset \omega^{\omega} \rightarrow \omega^{\omega}$ there exists a $\Gamma$-recursive function $\tilde{f}: \tilde{D} \subset \omega^{\omega} \times \omega^{\omega} \rightarrow \omega^{\omega}$ with $\tilde{D}(\varepsilon) \supset D$ such that $f(x)=\tilde{f}(\varepsilon, x)$ for all $x \in D$.

Observe that one cannot a priori replace in the arguments of Corollary 4.12 the substitution property by the weak substitution property.

Combining Theorem 4.2 and Theorem 4.9 we then get:

Corollary 4.13. If $\boldsymbol{\Gamma}$ is a non self-dual Wadge class containing the class $\boldsymbol{\Pi}_{1}^{0} \cup \boldsymbol{\Sigma}_{1}^{0}$ then the following are equivalent.

i) $\boldsymbol{\Gamma}$ is of level $\geq 2$.

ii) $\circlearrowright \boldsymbol{\Gamma}$ has the substitution property

iii) $\partial \boldsymbol{\Gamma}$ has the weak substitution property

\section{Norms AND ScAles on WAdGe Classes}

5.1. Norms: By a $\Gamma$-norm on a set $A$ we always mean a mapping $\varphi: A \rightarrow O n$ such that both relations:

$$
\left\{\begin{array}{l}
x \in A \text { and } \quad(y \notin A \text { or } \varphi(x) \leq \varphi(y) \\
x \in A \text { and } \quad(y \notin A \text { or } \varphi(x)<\varphi(y)
\end{array}\right.
$$

are in $\Gamma$.

Note that, though quite universal, this definition is not the one adopted in [7] where Moschovakis considers a weaker notion, which happens to be equivalent to the previous one if the class $\boldsymbol{\Gamma}$ is adequate (see [7]), or at least if $\boldsymbol{\Gamma}$ is closed under finite boolean operations. Unfortunately Moschovakis' definition is too weak to recover several of the fundamental properties of normed classes. On the other hand many normed Wadge classes are non adequate (see [6]), and for such classes working with the definition above is necessary. 
5.2. Scales: We recall that a $\boldsymbol{\Gamma}$-scale on a set $A \subset X$ is a sequence $\bar{\varphi}=\left(\varphi_{k}\right)_{k \in \omega}$ of $\boldsymbol{\Gamma}$-norms on $A$ satisfying:

$(\star)\left\{\begin{array}{l}\text { If a sequence }\left(x_{i}\right)_{i \in \omega} \text { in } A \text { is such that } x=\lim _{i} x_{i} \text { exists in } X \text { and for } \\ \text { each } k \text { and each } i \text { large enough } \varphi_{k}\left(x_{i}\right)=\xi_{k} \text { is constant, then } x \in A \text { and } \\ \text { for all } k, \varphi_{k}(x) \leq \xi_{k} .\end{array}\right.$

Note that in almost all significant uses of scales some additional properties are actually needed for the proofs. The most common ones are the following:

A scale $\bar{\varphi}$ will said to be:

a) good if any sequence $\left(x_{i}\right)_{i \in \omega}$ in $A$ such that for each $k$ and each $i$ large enough $\varphi_{k}\left(x_{i}\right)=\xi_{k}$ is constant, admits a limit in $X$.

b) monotonic if:

$$
\forall k \in \omega, \forall x, y \in A \text {, if } \varphi_{k+1}(x) \leq \varphi_{k+1}(y) \text { then } \varphi_{k}(x) \leq \varphi_{k}(y) .
$$

The scale will be said to be very good if it is good and monotonic.

In fact as far as one is working with adequate classes very good scales are easy to obtain. Indeed given any $\boldsymbol{\Gamma}$-scale $\left(\varphi_{k}\right)_{k \in \omega}$ on some set $A \subset \omega^{\omega}$ with $\varphi_{k}: A \rightarrow \lambda$, if we order $\hat{\lambda}_{k}=(\omega \times \lambda)^{k+1}$ lexicographically and define $\hat{\varphi}_{k}: A \rightarrow \hat{\lambda}_{k}$ by:

$$
\hat{\varphi}_{k}(x)=\left(x(0), \varphi_{0}(x), \cdots, x(k), \varphi_{k}(x)\right)
$$

then one can easily check that if the class $\boldsymbol{\Gamma}$ is adequate then $\left(\hat{\varphi}_{k}\right)_{k}$ is both good and monotonic. But this is no more true if $\boldsymbol{\Gamma}$ is not adequate. However as we shall see in this section if $\boldsymbol{\Gamma}$ is a any normed Wadge class of Borel sets one can still construct nice $\boldsymbol{\Gamma}$-scales for any set in $\boldsymbol{\Gamma}$. The proof of this result relies on results due to Louveau - Saint Raymond ([6]) as well as their proofs. For the ease of the reader we give first a brief presentation of these latter results.

In fact in [6] the authors characterize normed Wadge classes of Borel sets as exactly those which admit a Wadge description avoiding a prescribed number of Boolean set operations. This gives an internal inductive definition of these classes (see [6], Définition 16), and the main result of [6] is the following :

Theorem 5.3. (Louveau-Saint Raymond) For any Wadge class $\boldsymbol{\Gamma}$ the following are equivalent:

(i) $\boldsymbol{\Gamma}$ has the reduction property;

(ii) $\boldsymbol{\Gamma}$ is normed;

(iii) $\boldsymbol{\Gamma}$ is scaled.

However the scales in (iii) given by the proof of Theorem 5.3 in [6] are far away from being good or monotonic, and our main goal is to prove that this can actually be achieved. For this we need the following fundamental notion introduced in [6].

Definition 5.4. (Louveau-Saint RAYmond) Let $\boldsymbol{\Gamma}$ be a Wadge class of Borel sets and $A \subset \omega^{\omega}$ in $\boldsymbol{\Gamma}$. A family $\left(A_{n}, \xi_{n}\right)_{n \in \omega}$ is said to be a norming family for the set $A$ if:

(1) for all $n \in \omega, \xi_{n}<\omega_{1}$ and $A_{n} \in \mathbf{\Pi}_{\xi_{n}}^{0}$,

(2) $\left(A_{n}\right)_{n \in \omega}$ is a partition of $A$, 
(3) for any families $\left(C_{n}\right)_{n \in \omega}$ and $\left(V_{\xi}\right)_{\xi \in O n}$ of subsets of $\omega^{\omega} \times \omega^{\omega}$, if for all $n, \xi, C_{n} \in \Sigma_{\xi_{n}}^{0}$ and $V_{\xi} \in \Sigma_{\xi}^{0}$ then the set $\bigcup_{n \in \omega}\left(A_{n} \times \omega^{\omega}\right) \cap\left(C_{n} \backslash V_{\xi_{n}}\right)$ is in $\boldsymbol{\Gamma}$.

Theorem 5.5. If $\boldsymbol{\Gamma}$ is a normed Wadge class then any set in $\boldsymbol{\Gamma}$ admits a very good $\Gamma$-scale.

Proof. Let $A \in \boldsymbol{\Gamma}$; then by [6] (Théorème 20) $A$ admits a norming family $\left(A_{n}, \xi_{n}\right)_{n \in \omega}$. Set $\lambda=\sup _{n}\left(\omega \cdot \xi_{n}+n\right)$ and let $\varphi: A \rightarrow \lambda$ be defined by:

$$
\varphi(x)=\omega \cdot \xi_{n}+n \quad \text { if } x \in A_{n} .
$$

By a classical result of Kuratowski ([4], §37.II Corollary 1a) for all $n \in \omega$, setting $\xi_{n}=1+\xi_{n}^{*}$, we can fix a continuous bijection $f_{n}: \omega^{\omega} \rightarrow \omega^{\omega}$ such that $g_{n}:=f_{n}^{-1}$ : $\omega^{\omega} \rightarrow \omega^{\omega}$ is of class $\xi_{n}^{*}$ and $F_{n}=g_{n}(A)=f_{n}^{-1}(A) \in \mathbf{\Pi}_{1}^{0}$ (see also [1] for a much stronger version).

Then for all $k \in \omega$, let $\varphi_{k}: A \rightarrow \lambda \times \omega^{k}$ be defined by

$$
\varphi_{k}(x)=\left(\varphi(x), g_{n}(x)_{\mid k}\right) \quad \text { if } x \in A_{n}
$$

and set:

$$
B_{k}^{\prime}:=\left\{(x, y) \in \omega^{\omega} \times \omega^{\omega}: x \in A \text { and }\left(y \notin A \text { or } \varphi_{k}(x) \leq \varphi_{k}(y)\right)\right\} .
$$

where $\leq$ refers to the lexicographical ordering on $\lambda \times \omega^{k}$.

Claim 5.6. $B_{k}^{\prime} \in \boldsymbol{\Gamma}$

Proof: For all $n \in \omega$ and all $\xi \in O n$ the sets

$$
C_{n}^{\prime}=\bigcap_{\substack{m<n \\ \xi m=\xi_{n}}} \omega^{\omega} \times\left(\omega^{\omega} \backslash A_{m}\right) \quad \text { and } \quad V_{\xi}=\omega^{\omega} \times \bigcup_{\substack{m \in \omega: \\ \xi m<\xi}} A_{m}
$$

are clearly $\boldsymbol{\Sigma}_{\xi_{n}}^{0}$ (resp. $\boldsymbol{\Sigma}_{\xi}^{0}$ ) subsets of $\omega^{\omega} \times \omega^{\omega}$. Also since for all $u \in \omega^{k}$ the set $g_{n}^{-1}\left(N_{u}\right)=\left\{x \in \omega^{\omega}: g_{n}(x)_{\mid k}=u\right\}$ is $\boldsymbol{\Delta}_{\xi_{n}}^{0}$ then

$$
D_{n}=\left\{(x, y): g_{n}(x)_{\mid k} \leq g_{n}(y)_{\mid k}\right\}=\bigcup_{\substack{u \leq v \\ u, v \in \lambda \times \omega^{k}}}\left\{x: g_{n}(x)_{\mid k}=u\right\} \times\left\{y: g_{n}(y)_{\mid k}=v\right\}
$$

is a $\Sigma_{\xi_{n}}^{0}$ subset of $\omega^{\omega} \times \omega^{\omega}$ too, and so are the sets $C_{n}^{\prime \prime}=D_{n} \cup\left(\omega^{\omega} \times\left(\omega^{\omega} \backslash A_{n}\right)\right)$ and $C_{n}=C_{n}^{\prime} \cap C_{n}^{\prime \prime}$. Hence by Definition 5.4 the set $B_{k}=\bigcup_{n \in \omega}\left(A_{n} \times \omega^{\omega}\right) \cap\left(C_{n} \backslash V_{\xi_{n}}\right)$ is in $\boldsymbol{\Gamma}$, and we now show that $B_{k}=B_{k}^{\prime}$.

Observe first that $A \times\left(\omega^{\omega} \backslash A\right) \subset B_{k} \subset A \times \omega^{\omega}$ and $A \times\left(\omega^{\omega} \backslash A\right) \subset B_{k}^{\prime} \subset A \times \omega^{\omega}$ so we only need to show that $B_{k} \cap(A \times A)=B_{k}^{\prime} \cap(A \times A)$. So fix $(x, y) \in A \times A$; then $x \in A_{n}$ and $y \in A_{p}$ for some uniquely determined $n, p$.

If $(x, y) \in B_{k}$ then since $y \in A_{p}$ and $(x, y) \in C_{n}^{\prime} \backslash V_{\xi_{n}}$ then either $\xi_{p}>\xi_{n}$ or $\left(\xi_{p}=\xi_{n}\right.$ and $\left.p \geq n\right)$, and in both cases $\varphi(x) \leq \varphi(y)$ :

- if $\varphi(x)<\varphi(y)$ then $\varphi_{k}(x)<\varphi_{k}(y)$, so $(x, y) \in B_{k}^{\prime}$;

- if not then $\varphi(x)=\varphi(y)$ hence $m=n$ so $y \in A_{n}$, and since $(x, y) \in C_{n}^{\prime \prime}$ then $y \in D_{n}$ so $g_{n}(x)_{\mid k} \leq g_{n}(y)_{\mid k}$, hence $\left.\varphi_{k}(x) \leq \varphi_{k}(y)\right)$ and $(x, y) \in B_{k}^{\prime}$. 
Conversely if $(x, y) \in B_{k}^{\prime}$ then $\varphi_{k}(x) \leq \varphi_{k}(y)$, in particular:

$$
\omega \cdot \xi_{n}+n=\varphi(x) \leq \varphi(y)=\omega \cdot \xi_{p}+p .
$$

Then for all $m$, if either $m<n$ is such that $\xi_{m}=\xi_{n}$, or if $\xi_{m}<\xi_{n}$, then $\omega \cdot \xi_{m}+m<\varphi(y)=\omega \cdot \xi_{p}+p$, and it follows that $y \notin A_{m}$; hence $(x, y) \in C_{n}^{\prime} \backslash V_{\xi_{n}}$. - If $p \neq n$ then $y \notin A_{n}$ so $(x, y) \in C_{n}^{\prime \prime}$, hence $(x, y) \in C_{n} \backslash V_{\xi_{n}}$.

- If $p=n$ then $y \in A_{n}$ and $\varphi(x)=\varphi(y)$, and since $\varphi_{k}(x) \leq \varphi_{k}(y)$ then $g_{n}(x)_{\mid k} \leq$ $g_{n}(y)_{\mid k}$, so $(x, y) \in D_{n}$ and again $(x, y) \in C_{n} \backslash V_{\xi_{n}}$.

Then since $x \in A_{n}$ then in both cases $(x, y) \in B_{k}$.

This finishes the proof of the claim.

In a similar way one can prove that for all $k$ the set

$$
B_{k}^{\prime \prime}:=\left\{(x, y) \in \omega^{\omega} \times \omega^{\omega}: x \in A \text { and }\left(y \notin A \text { or } \varphi_{k}(x)<\varphi_{k}(y)\right)\right\}
$$

is in $\boldsymbol{\Gamma}$ too. Hence if $\theta_{k}$ is any embedding of $\lambda \times \omega^{k}$ in $\omega_{1}$ then $\psi_{k}=\theta_{k} \circ \varphi_{k}$ is a $\Gamma$-norm on $A$.

Suppose now that a sequence $\left(x_{i}\right)_{i \in \omega}$ in $A$ is such that $x=\lim _{i} x_{i}$ exists in $\omega^{\omega}$ and that for all $k$ and all $i \geq i_{k}, \varphi_{k}\left(x_{i}\right)=\mu_{k}$ is constant. In particular for all $i \geq i_{0}, \varphi\left(x_{i}\right)=\omega \cdot \xi_{n}+n$ is constant, that is $x_{i} \in A_{n}$. Moreover for all $k$, if $g_{n}^{(k)}$

denotes the $k^{\text {th }}$ coordinate of $g_{n}$, then $\lim _{i} g_{n}^{(k)}\left(x_{i}\right)$ exists, hence $\lim _{i} g_{n}\left(x_{i}\right)=y$ exists. Since $F_{n}=g_{n}\left(A_{n}\right)$ is closed and $g_{n}\left(x_{i}\right) \in F_{n}$ for all $i \geq i_{0}$, then $y \in F_{n}$ and so $f_{n}(y) \in A_{n}$; and since $f_{n}=g_{n}^{-1}$ is continuous then

$$
x=\lim _{i} x_{i}=\lim _{i} f_{n}\left(g_{n}\left(x_{i}\right)\right)=f_{n}(y) \in A_{n}
$$

Moreover for all $k$ and all $i \geq i_{k}$, we have:

$$
\varphi_{k}(x)=\left(\varphi(x), g_{n}(x)_{\mid k}\right)=\left(\varphi(x), y_{\mid k}\right)=\left(\varphi(x), g_{n}\left(x_{i}\right)_{\mid k}\right)=\varphi_{k}\left(x_{i}\right)=\mu_{k} .
$$

This proves that the sequence $\left(\psi_{k}\right)_{k \in \omega}$, which is obviously monotonic, is also a good $\Gamma$-scale on $A$.

Remark 5.7. As one can easily check the scale $\left(\psi_{k}\right)_{k \in \omega}$ constructed in the previous proof satisfies the following additional "continuity" property:

$(\star \star)\left\{\begin{array}{l}\text { If a sequence }\left(x_{i}\right)_{i \in \omega} \text { in } A \text { is such that } x=\lim _{i} x_{i} \text { exists in } X \text { and for } \\ \text { each } k \text { and each } i \text { large enough } \psi_{k}\left(x_{i}\right)=\xi_{k} \text { is constant, then } x \in A \text { and } \\ \text { for all } k, \psi_{k}(x)=\xi_{k} .\end{array}\right.$

\section{The game operator on NORMed WAdGe Classes}

Most fundamental results on $\partial \Gamma$ are due to Moschovakis. Unfortunately all these results are stated and proved in [7] under the assumption that the class $\Gamma$ is adequate class. However, inspecting the proofs one can observe that in each case the adequateness assumption on $\Gamma$ is only used to reformulate the hypothesis and is no more needed in the core of the proof. More precisely we can restate Theorem 6D.3 and Theorem 6E.1 of [7] as follows: 
Theorem 6.1. (Moschovakis) Let $\Gamma$ be a class, and assume that Det $(\boldsymbol{\Gamma})$ holds where $\boldsymbol{\Gamma}=\bigcup_{\varepsilon \in \omega^{\omega}} \Gamma(\varepsilon)$.

a) If $\Gamma$ is normed then $\partial \Gamma$ is normed.

b) If the set $A \subset \omega^{\omega} \times \omega^{\omega}$ admits a monotonic $\Gamma$-scale then there exists $a \supset \Gamma$ recursive function which assigns to any element $x \in \partial A$ a winning strategy for Player I in the game $\mathbf{G}_{A(x)}$.

In fact for part a) the adequateness assumption on $\Gamma$ in [7] (Theorem 6D.3) is used to ensure that any $\Gamma$-norm (in the weak sense of [7]) is automatically a $\Gamma$-norm (in the sense of Section 5.1). Then the rest of the proof of Theorem 6D.3 associates explicitly to any $\Gamma$-norm on a set $A \subset X \times \omega^{\omega}$, a $\supset \Gamma$-norm on the set כA .

Also part b) is stated in [7] (Theorem 6E.1) under the (weaker) hypothesis that the set $A$ admits an arbitrary $\Gamma$-scale, but again assuming $\Gamma$ to be adequate. In this case the adequateness assumption is used to construct first a very good $\Gamma$-scale $\bar{\varphi}$ for the set $A$, but as one can check only the monotonicity of $\bar{\varphi}$ is used in the rest of the proof.

Combining Theorem 5.5 and Theorem 6.1 we get:

Theorem 6.2. If $\boldsymbol{\Gamma}$ is a normed Wadge class of Borel sets then:

a) $\partial \boldsymbol{\Gamma}$ is normed.

b) For any set $A \subset \omega^{\omega} \times \omega^{\omega}$ in $\boldsymbol{\Gamma}$ there exists a $\partial \boldsymbol{\Gamma}$-recursive function which assigns to any $x \in \partial A$ a winning strategy for Player I in the game $\mathbf{G}_{A(x)}$.

We now give two applications. The first application relies on the main result of [2] which asserts that if the class $\partial \boldsymbol{\Gamma}$ satisfies conditions a) and b) of Theorem 6.2 and has moreover the substitution property then $\partial \boldsymbol{\Gamma}$ satisfies the conclusion of Corollary 6.3, which generalizes a result of Kechris ([3]) for the class $\boldsymbol{\Pi}_{1}^{1}=\partial \boldsymbol{\Sigma}_{1}^{0}$.

Let us recall that given a class $\boldsymbol{\Lambda}$, a set $A$ is said to be $\boldsymbol{\Lambda}$-hard if any set $B \in \boldsymbol{\Lambda}$ is reducible to $A$ by a continuous function that is if there is some continuous function $\varphi: 2^{\omega} \rightarrow X$ such that $B=\varphi^{-1}(A)$.

Corollary 6.3. Let $\boldsymbol{\Gamma}$ be a normed Wadge class of level $\geq 2$, and $A$ a subset of some Polish space $X$. If any. $\mathrm{\Gamma} \boldsymbol{\Gamma}$-set is reducible to $A$ by a $\partial \boldsymbol{\Gamma}$-measurable function then $A$ is $\supset \boldsymbol{\Gamma}$-hard.

Proof. If $X=\omega^{\omega}$ then it follows from Theorems 4.9 and 6.2 that all the assumptions of Theorem 1.4 in [2] are satisfied; hence $A$ is $\partial \boldsymbol{\Gamma}$-hard. For the general case fix any continuous bijection $f$ from $\omega^{\omega}$ onto $X$; then $f^{-1}: X \rightarrow \omega^{\omega}$ is automatically Borel. Note that if a set $B \subset 2^{\omega}$ is reducible to $A$ by a $\supset \boldsymbol{\Gamma}$ measurable function $\varphi: 2^{\omega} \rightarrow X$, then $B$ is reducible to $f^{-1}(A)$ by the function $f^{-1} \circ \varphi: 2^{\omega} \rightarrow \omega^{\omega}$ which is $\partial \boldsymbol{\Gamma}$-measurable too, since the class $\partial \boldsymbol{\Gamma}$ is closed under countable unions and intersections (see [7], Theorem 6D.2). It follows then from the particular case $X=\omega^{\omega}$ that $f^{-1}(A)$ is $\partial \boldsymbol{\Gamma}$-hard. Hence any set $B \subset 2^{\omega}$ in $\partial \boldsymbol{\Gamma}$ is reducible to $f^{-1}(A)$ by a continuous function $\psi: 2^{\omega} \rightarrow X$, so $B$ is reducible to $A$ by the continuous function $f \circ \psi: 2^{\omega} \rightarrow X$, which proves that $A$ is $\circlearrowright \boldsymbol{\Gamma}$-hard. 
The second application concerns Spector classes which are introduced and studied in [7]. We recall that a class $\Sigma$ is said to be a Spector class if:

1) $\Sigma$ is $\omega$-parametrized and closed under recursive substitutions, $\exists^{\omega}$ and $\forall^{\omega}$,

2) $\Sigma$ is normed,

3) $\Sigma$ has the substitution property.

In fact the only known Spector classes are all of the form $\partial \Gamma$. Note that in this case condition 1) is satisfied for any $\omega$-parametrized class $\Gamma$. By a result of Kechris and Moschovakis ([7], Theorem 6D. 4) $\partial \Sigma_{n}^{0}$ is a Spector class for all $n \in \omega$; but the arguments extend easily to $\partial \Sigma_{\xi}^{0}$ for all $1 \leq \xi<\omega_{1}^{C K}$. We also recall that in [6] it is proved that for all $1 \leq \eta, \xi<\omega_{1}$, the class $D_{\eta}\left(\Sigma_{\xi}^{0}\right)$ is normed, and by a straightforward adaptation of the arguments one can show that the corresponding effective class $D_{\eta}\left(\Sigma_{\xi}^{0}\right)$ is also normed. Then it follows from Theorem $6.2 \mathrm{a})$ that the class $\partial\left(D_{\eta}\left(\Sigma_{\xi}^{0}\right)\right)$ is normed. Hence, aside the case $\eta=1$ for which $\partial\left(D_{1}\left(\Sigma_{\xi}^{0}\right)\right)=\partial \Sigma_{\xi}^{0}$ is always a Spector class, for the class $\partial\left(D_{\eta}\left(\Sigma_{\xi}^{0}\right)\right)$ with $\eta \geq 2$, being Spector is equivalent to have the substitution property, which by Theorem 4.2 and Corollary 4.12 is equivalent to be of level $\geq 2$.

Corollary 6.4. For all $2 \leq \eta, \xi<\omega_{1}^{C K}$,

a) $\partial\left(D_{\eta}\left(\Sigma_{\xi}^{0}\right)\right)$ is a Spector class.

b) $\partial\left(D_{\eta}\left(\Sigma_{1}^{0}\right)\right.$ is not a Spector class.

Let us also mention without proof that following similar arguments one can prove that if $\boldsymbol{\Gamma}$ is a $\Delta_{1}^{1}$-Wadge class in the sense of [5] and $\Gamma=\boldsymbol{\Gamma} \cap \Delta_{1}^{1}$ then the class $\partial \Gamma$ is a Spector class if and only if either $\partial \Gamma=\Pi_{1}^{1}$ or $\boldsymbol{\Gamma}$ is a normed Wadge class of level $\geq 2$, in which case $\partial \Gamma \supset \partial \Sigma_{2}^{0}$. An exhaustive proof of this necessitates to resume completely the content of [6] and give effective versions of all the results.

\section{REFERENCES}

1. G. Debs and J. Saint Raymond, Borel liftings of Borel sets: Some decidable and undecidable statements,. Memoirs of the Amer. Math. Soc. 187 (2007), no. 876. MR2308388.

2. G. Debs and J. Saint Raymond, On $\mathrm{D} \boldsymbol{\Gamma}$-complete sets. To appear.

3. A. Kechris, On the concept of $\Pi_{1}^{1}$-completeness. Proc. Amer. Math. Soc. 125 (1997), no. 6, 1811-1814.

4. K. Kuratowski, Topology Vol. I. New edition, revised and augmented. Translated from the French by J. Jaworowski Academic Press, New York-London; Państwowe Wydawnictwo Naukowe, Warsaw (1966).

5. A. Louveau, Some results in the Wadge hierarchy of Borel sets: The Cabal Seminar, vol. II. Lecture Notes in Logic. vol. 37, Cambridge University Press 2012.

6. A. Louveau and J. Saint Raymond, les propriétés de réduction et de norme pour les classes de Boréliens. Fund. Math. 131 (1988), 223-243.

7. Y. N. Moschovakis, Descriptive Set Theory, Studies in logic 100 North-Holland, Amesterdam (1979); second edition: Mathematical surveys and monographs 155, Amer. Math. Soc. Providence, Rhode Island (2009) 
Gabriel Debs, Sorbonne Université, Université Paris Diderot, CNRS, Institut de Mathématiques de Jussieu-Paris Rive Gauche, IMJ-PRG, F-75005, Paris, France, and Université Le Havre Normandie, Institut Universitaire de Technologie, Rue Boris Vian, BP 400676610 Le Havre, France.

E-mail address: gabriel.debs@imj-prg.fr

Jean Saint Raymond, Sorbonne Université, Université Paris Diderot, CNRS, Institut de Mathématiques de Jussieu-Paris Rive Gauche, imJ-PRG, F-75005, Paris, FRANCE

E-mail address: jean.saint-raymond@imj-prg.fr 